\title{
RESUMEN
}

Las trasmisiones radifónicas y televisivas de carácter religioso probablemente ejercen su eficacia según las reglas que se han podido constatar para la eficacia de trasmisiones semejantes. Un importante resultado parcial de las observaciones hechas en materia de eficacia, y que vale también para las trasmisiones religiosas, es la siguiente: Lo que es popular no convierte; lo que ha de tener eficacia de conversión no ha de ser popular. Sin embargo, las trasmisiones eclesiásticas han de aspirar a una popularidad en vista de aquellos cristianos que se mantienen alejados de la Iglesia. Pues en amplias esferas se ha mantenido un fondo religioso. Se debe llegar a estas esferas por medio de un lenguaje actual para el cual tengan interés. Para ello se ha de elegir una conveniente estrategia de comunicationes: es diverso el lenguaje adecuado a trabajadores que el propio para intelectuales. A ambos grupos se ha de presentar los dos lados de los temas controvertidos para que puedan llegar a una conclusión personal. La eficacia de las trasmisiones eclesiásticas puede aquilatarse si, con mucho tacto, se toma en cuenta las disposiciones del público. En tal esfuerzo, sin embargo, téngase en cuenta la exhortación del apóstol San Pablo: "Predica la palabra, insiste a tiempo y destiempo..." (2 Tim. 4.2).

\section{Publizistik im Spiegel von Konzilsdekret und Uppsala-Erklärung}

\author{
von Heinz Melzer
}

Das Dekret über die sozialen Kommunikationsmittel „Inter Mirifica ${ }^{*}$ des II. Vatikanischen Konzils, am 4. Dezember 1963 feierlich verkündet, hat von allen Konzilsdokumenten wohl die heftigste Kritik erfahren. Jedoch hat sich im Laufe der Jahre auch herausgestellt, daß dieses Dekret nicht nur keinen Abschluß der Diskussion über seinen Gegenstand bedeutete, sondern daß es diese Debatte erst richtig in Gang gebracht hat. Das zeigte sich auch während des 8. Weltkongresses der Katholischen Weltunion der Presse Anfang Juli (2.-6.) 1968 in Berlin, auf dem der Wiener Erzbischof Kardinal König feststellte, das Dokument „Inter Mirifica“ wäre gewiß besser ausgefallen, wenn es zu einem späteren Zeitpunkt des Konzils diskutiert worden wäre, etwa nach der Pastoralkonstitution über die "Kirche in der Welt von heute", in deren Licht es interpretiert werden müsse. ${ }^{1}$ Auf die Notwendigkeit dieser Interpretation weist schon das kleine Konzilskompendium hin: „Folgt man

Heinz Melzer ist Leiter der Pressestelle der Evangelischen Arbeitsgemeinschaft für Weltmission und des Deutschen Evangelischen Missionsrates in Hamburg. Der Text der hier behandelten Uppsala-Erklärung über „Die Kirche und die Medien der Massenkommunikation" wird in seiner endgültigen Fassung als Anhang XI im Abschlußbericht der 4. Vollversammlung des Okumenischen Rates der Kirchen veröffentlicht, der noch 1968, herausgegeben von Norman Goodall unter dem Titel "Bericht aus Uppsala 1968“ im Selbstverlag des ORK (Editions Oikoumene), Genf, erscheinen soll. Der Autor stützt sich in seinen hier verwendeten Zitaten noch auf die Entwurfsfassung. Das Dokument ist auch veröffentlicht in "Medium “, München, 5. Jg. 1968, S. 196-214. 
der Grundintention des Konzils, so ist das Dekret gemäß der dogmatischen Konstitution über die Kirche und nach der Pastoralkonstitution über die Kirche in der Welt von heute $\mathrm{zu}$ interpretieren".2 Positiv ist auf jeden Fall zu bewerten, daß die römisch-katholische Kirche in diesem Dekret überhaupt erstmalig Fragen der Massenmedien eine „eigene und relativ sorgfältige Beachtung geschenkt hat ${ }^{\alpha}{ }^{3}{ }^{3}$

Im Juli 1968 wurde dem Ókumenischen Rat der Kirchen auf seiner 4. Vollversammlung in Uppsala (4.-19. Juli) eine Erklärung über "Die Kirche und die Medien der Massenkommunikation" (Fernsehen, Rundfunk, Nachrichtensatelliten, Filme, Zeitungen und Zeitschriften, Schallplatten, Bücher und Tonbänder) vorgelegt und "in ihrem wesentlichen Inhalt gebilligt und den Kirchen zum Studium und zu entsprechender Veranlassung empfohlen". Dieses Dokument, das sich an die Kirchen richtet, die "für die Medien mitverantwortlich" sind, sagt in seiner Selbstbeurteilung, es wolle "nur ein Anfang" sein, da die Welt der Massenkommunikationsmittel „so neu und oft so verwirrend für die Kirchen [ist], daß wir gerade erst ihre Bedeutung, ihre Möglichkeiten, Entstellungen [im englischen Text: perversions] und endgültige Verwendungsarten zu sondieren beginnen". Die Erklärung soll nach Penry Jones, dem Leiter der Religiösen Abteilung der Londoner BBC, der das Dokument der Vollversammlung erläuterte, ein vernünftiges Alpha sein, „ohne den Versuch, es bis zum Omega zu füllen. Das Dokument ist in gar keiner Weise ein Dekret". Es sei zu hoffen, daß der Entwurf den Kirchen sinnvoll erscheine, ohne den "Professionellen" sinnlos zu erscheinen.

Die Uppsala-Erklärung über die Massenkommunikationsmittel will gewiß als ein Beitrag zum ökumenischen Dialog verstanden werden. Deshalb sollen einige ihrer Aussagen gegen die Aussagen des Dekrets "Inter Mirifica“ abgewogen werden. Es wird hier kein vollständiger Textvergleich angestrebt, sondern nur einige Punkte, an denen die Diskussion weitergeführt werden könnte, sollen aufgezeigt werden.

Die Erklärung des Ókumenischen Rates über die Massenmedien ist das Ergebnis mehrerer Konsultationen. Unter Vorsitz des hannoverschen Landesbischofs D. Dr. Hanns Lilje kamen in den vergangenen Jahren Publizisten aus der säkularen und kirchlichen Presse zu diesen Konsultationen zusammen. Die Verfasser hielten es für gut, Fernsehen und Hörfunk einen besonders breiten Raum zu geben. Das ist verständlich, denn hier sind zugegebenermaßen die Probleme, die sich zwischen den vorgegebenen Strukturen einerseits und dem menschlichen Willen auf der anderen Seite auftun, am schwierigsten zu lösen. Dazu Penry Jones: „Wenn der Erzengel Michael selbst leitender Direktor einer Fernsehgesellschaft wäre und wenn die sechs Präsidenten des Okumenischen Rates seine Betriebsratsvorsitzenden wären, so würde es immer noch Probleme geben. ${ }^{\text {" }}$

Die Uppsala-Erklärung über die Medien der Massenkommunikation setzt nicht mit theologischen Reflexionen, sondern, nach einem kurzen Vorwort, bei den Massenmedien selbst ein und untersucht zunächst die Auswirkungen der "Revolution der Kommunikationsmittel " auf Gesellschaft und Kirche in einer kleiner werdenden Welt. Erst in einem zweiten Teil folgen theologische Bemerkungen, im dritten Teil schließlich werden Empfehlungen an die Kirchen ausgesprochen.

Sowohl bei dem Dekret "Inter Mirifica" als auch in der Uppsala-Erklärung über die Medien der Massenkommunikation wird die Schwierigkeit deutlich, „global* zu sprechen, da die Situationen der Kirchen und die Strukturen und Entwricklungsstadien der Massenmedien in den verschiedenen Regionen der Erde und in den verschiedenen Ländern sich keinesfalls auf einen Nenner bringen lassen.

"Inter Mirifica“ stellt das ursprüngliche Recht der Kirche, jedes dieser sozialen 
Kommunikationsmittel „zu benutzen und zu besitzen, soweit es für die christliche Erziehung und ihr Wirken am Heile der Seelen notwendig und nützlich ist ${ }^{\text {", }}$ fest. $^{5}$ Dieses Recht wird sich in kirchenfeindlichen Gesellschaften nicht durchsetzen lassen. Die Uppsala-Erklärung geht auf die "unterschiedliche Einstellung, bedingt durch die politische Struktur des Landes oder durch Wesen und Ausmaß der Kontrolle über diese Medien" ein und sagt, daß die Kirchen in einer so komplexen Situation gut daran täten, „ihr traditionelles Mißtrauen gegenüber den Medien aufzugeben und eine feste Partnerschaft mit denjenigen einzugehen oder zu suchen, die die modernen Kommunikationssysteme schaffen, erstellen, gebrauchen und bewerten“.

Das Konzilsdekret stellt als Hauptmotive, die die Kirche zur Beschäftigung mit den Kommunikationsmitteln bewegen, einerseits die Sorge heraus, daß mit Hilfe dieser Medien so große Macht und so großer Einfluß auf die Gesellschaft ausgeübt und daß sie zum Schaden und Unheil der Menschen mißbraucht werden können; andererseits betont das Dokument, daß die Kirche diese einflußreichen Mittel für ihre Aufgabe, die Ausbreitung und Festigung des Gottesreiches, benutzen sollte. Diese beiden Motive klingen auch in der Uppsala-Erklärung an, jedoch wird hier sehr viel stärker die positive Wirkung der Massenmedien betont und gesagt, daß die Medien es den Menschen wie niemals zuvor ermöglichen, ihre Erfahrungen zu teilen „in der Hoffnung, daß die Menschen in wachsendem Maße erkennen, verstehen und mitfühlen. Die Medien liefern einige Grundstrukturen für eine verantwortliche Weltgesellschaft. Die Leiden anderer werden rasch bekannt und können schnell gelindert werden. Die wesentlichen Probleme unserer Zeit werden von allen Menschen diskutiert. Minderheitenansichten können öffentlich vertreten werden." Diese Aussage scheint fast $z u$ optimistisch. Aber es wird auch gesagt, daß die Medien zwar diese Dinge bewirken können, jedoch gebe es dafür keine Garantie. "Während die Massenkommunikationsmittel den Geschmack und die Urteilskraft verbessern können, ist es ebensogut möglich, daß sie die Kultur auf einem äußerst niedrigen Niveau erstarren lassen." Trotz aller dieser Vorbehalte bleibt der Grundton der Uppsala-Erklärung gegenüber den Massenmedien positiv. Dieser Ton klingt im letzten Satz des Dokumentes noch einmal stark auf: „damit wir mit allen Menschen guten Willens zur Humanisierung unserer Gesellschaft beitragen und damit so das Amt der Kirche für ein wirksameres Zeugnis vor allen Menschen erneuert wird." Mit diesem Ziel gibt die Uppsala-Erklärung der Hoffnung Ausdruck, daß die Kirchen und der Okumenische Rat der Kirchen die in dieser Erklärung begonnene Diskussion fortsetzen.

Der Gedanke, „das vielgestaltige Apostolatswerk der Kirche auf dem Gebiet der sozialen Kommunikationsmittel wirksam zu kräftigen ", zieht sich wie ein roter Faden durch das Dekret „Inter Mirifica“, und auch die Uppsala-Erklärung sagt, $\mathrm{daß}$ die „mit den Medien verbundene missionarische Aufgabe riesige Dimensionen hat". "Diese Mission geschieht, wo immer Christen bei der Arbeit in den Medien mit allen Menschen guten Willens zusammenarbeiten, um die Mächte zu Dienern zu machen, d. h. für eine Rolle und Funktion der Medien zu arbeiten, die das Entstehen und Wachsen einer verantwortlichen Gesellschaft möglich machen. In diesem Lichte können die Medien als potentielle Werkzeuge der Mission gesehen werden." Dabei komme es vor allem auf die hohe Qualität der Darbietung, eine von allen verstandene Sprache und die Achtung vor Menschen anderen Glaubens oder ohne Glauben an. „Werden diese Kriterien nicht beachtet, wirkt der Gebrauch der Medien gegen die Mission Gottes." 
Bezeichnenderweise stehen diese Ausführungen über die großartigen Möglichkeiten, die die Massenkommunikationsmittel für die Mission bieten, nicht am Beginn, sondern am Ende der theologischen Bemerkungen der Uppsala-Erklärung. Die Erkenntnis, daß Kommunikation die Art ist, „in der Gott sich dem Menschen zu erkennen gibt und in der der Mensch Gott antwortet" ${ }^{\text {, }}$ ist die Kernaussage des theologischen Abschnittes der Uppsala-Erklärung. Aus dieser umfassenden Schau, die den ganzen Menschen in seiner Gemeinschaft als für Gott beansprucht sieht, ergibt sich, daß für die Beziehung zwischen Kirche und Medien nicht nur der Gebrauch, "sondern auch die Struktur und die Funktion der Medien das volle Engagement der christlichen Gemeinschaft erfordern". Das missionarische Anliegen der Kirche umfaßt nach der Uppsala-Erklärung „die gesamte Struktur und Verwendung der Massenmedien und nicht nur den kleinen Ausschnitt, für den die Kirchen unmittelbar verantwortlich sind". Mit anderen Worten: Die Kirche muß sich mit dem ganzen Gebiet der Massenmedien um seiner selbst willen befassen und nicht allein auf die missionarische Verwertbarkeit blicken.

Völlig einig sind sich, und das ist fast banal zu sagen -, überblickt man die großen Organisationen, durch die die Arbeit der Massenmedien überhaupt erst möglich wird -, muß aber wohl stets neu und nachdrücklich betont werden, das Dekret "Inter Mirifica ${ }^{\alpha}$ und die Uppsala-Erklärung in der Erkenntnis, daß der erforderliche finanzielle Aufwand für die Massenkommunikationsmittel sehr groß ist. Die Uppsala-Erklärung denkt in diesem Zusammenhang auch an die notwendige finanzielle Hilfe, die auf dem Gebiet der Massenkommunikationsmittel für wirtschaftlich arme Länder und Minderheitengruppen erforderlich ist.

Dem Dekret "Inter Mirifica " ist mehrfach der Vorwurf gemacht worden, es sei in seinem Grundtenor und in seiner Grundhaltung von einem gewissen Klerikalismus nicht freizusprechen. Das wird u. a. auch damit - aber nicht nur damit begründet, daß in diesem Dekret nur an einer einzigen Stelle (Artikel 3) die eigenständige Bedeutung der Laien und ihrer weltlichen Aufgabe angedeutet ist. Bei der Erwähnung der Laien im Artikel 13 handelt es sich um eine nachträgliche Einfügung auf Grund der Diskussion, „die am Tenor des Ganzen nicht viel ändert". ${ }^{\circ}$ Jedoch scheint einer der Hauptgründe für den Vorwurf des Klerikalismus zu sein, daß „Inter Mirifica“ zuviel von den Rechten und zu wenig von den Pflichten der Kirche spricht. Die Uppsala-Erklärung hingegen wurde als „energische Absage an klerikale Engstirnigkeit" begrüßt. In der Tat läßt der Text einen Verzicht auf Kirchenenge erkennen, schon deshalb, weil die Erklärung die spezifisch kirchlichen Sendungen (in Hörfunk und Fernsehen) nur am Rande behandelt und dann auch unter zwei entscheidend wichtigen Gesichtspunkten: Kirchliche Sendungen sollen mediengerecht sein und sie sollen sich nicht "billiger Werbemethoden" bedienen, die als "Propaganda“ zu verstehen seien? ${ }^{7}$. Der Gebrauch der Medien für die Verkündigung ist sicherlich eine gute Sache, aber sie ist unbedeutend im Vergleich zum Anspruch auf das Ganze der Medien, die dem ganzen Menschen dienen sollen, für Christus.

Während in "Inter Mirifica“ das Recht auf Information als natürliches Recht des Menschen bezeichnet wird und also die Informationsfreiheit begründet, wird die Frage, wie weit das Recht auf Informationen auch in der Kirche gilt, überhaupt nicht gestellt. Hier muß gesehen werden, daß die Struktur der römisch-katholischen Kirche nicht demokratisch, sondern hierarchisch ist und daß es sich bei dem Recht auf Information in der Kirche nicht "um ein dem demokratischen Recht auf Information paralleles und absolut gleichartiges Recht handelt “. ${ }^{8}$ Die Uppsala-Erklärung 
stellt fest, daß die Kirchen selbst in ihrer Informationspolitik und -praxis die Verpflichtung haben, "Informationen frei zugänglich zu machen. Außer in Bereichen wie etwa der privilegierten Kommunikation der Beichte sollten sich die Kirchen nicht scheuen, ihr eigenes Leben der öffentlichen Kritik auszusetzen. Die Kirchen dürfen nicht auf andere Institutionen Prinzipien anwenden, die sie in ihren eigenen Angelegenheiten nicht $\mathrm{zu}$ praktizieren bereit sind."

"Inter Mirifica“ fordert im Artikel 14 die Förderung der guten Presse. „Um jedoch die Leser ganz mit christlichem Geist zu erfüllen, soll auch eine katholische Presse gegründet und gefördert werden, die diesen Namen wirklich verdient.“ Träger dieser Presse sollen entweder die Kirche selbst oder katholische Persönlichkeiten sein. Es scheint in der Begründung dieser Forderung „eine gewisse Form von Triumphalismus zum Ausdruck zu kommen, die sicher nicht im Geiste des Konzils ist. Damit ist nichts gegen die Notwendigkeit und den großen Nutzen einer katholischen Presse gesagt, die aber sehr wohl viel bescheidener begründet und gerechtfertigt werden müßtec, urteilt ein Kritiker des Dekrets. ${ }^{9}$ An Bescheidenheit fehlt es der Uppsala-Erklärung gewiß nicht. So wird festgestellt, daß die Kirchen als Produzenten und in manchen Ländern auch als Eigentümer von Medien fungieren. "Zu oft benutzen sie diese Kanäle hauptsächlich als Erweiterung ihrer Kanzeln und Gemeindeblätter, und oft sind ihre Erzeugnisse, gemessen an professionellen Maßstäben, wertlos. Wenn sie sich auf andere als religiöse Kommunikation einlassen, liefern sie oft traurige Nachahmung dessen, was andere besser können. Es geht darum, ob die Kirchen sich selbst Maßstäbe für Qualität und Integrität setzen können, die als Ermutigung und Beispiel für die Kommunikationsindustrie ihres Landes dienen können." Wenn die Kritik des "Triumphalismus" das Konzilsdekret trifft, muß dem die Kritik an der Uppsala-Erklärung gegenübergestellt werden, daß in diesem Dokument „übertrieben viel Selbstanklage der Kirche“ zu finden sei und auch „ein zu großer Mangel an Selbstbewußtsein“, etwa wenn sich die Kirche damit einverstanden erklärt, in der pluralistischen Gesellschaft parallel mit Gewerkschaften, Parteien oder Vereinen gesetzt zu werden. „Demut sollte nicht mit Kleinmut, Bescheidenheit nicht mit Unterwerfung unter die Maßstäbe des Profanen verwechselt werden. ${ }^{{ }_{10}}$

Wenn in der Uppsala-Erklärung einerseits die Erkenntnis deutlich und ernst ausgesprochen wird, daß "die Medien der Massenkommunikation sowohl Produkt als auch Beschleuniger des Säkularisierungsprozesses sind ", der nicht unbedingt zum Säkularismus führen muß, sondern im wesentlichen einen Befreiungsprozeß von den "Ismen " darstellt, ${ }^{11}$ so wird doch auch nicht minder eindrücklich dargelegt, daß „der Beitrag der Massenmedien zum interkonfessionellen Verständnis für die ökumenische Bewegung ebenso wichtig [war] wie die Erfindung der Druckkunst für die Reformation. Verschiedene Denominationen mußten lernen, in den Rundfunkabteilungen und bei religiösen Programmen zusammenzuarbeiten ... Dieser Prozeß eines offenen und öffentlichen Dialogs, in dem bisher für selbstverständlich gehaltene Gruppenvorstellungen zur Debatte stehen, tragen dazu bei, von gegenseitiger Feindseligkeit zu einem größeren gemeinsamen Vokabular zu gelangen. Weiterführende Gemeinschaften überlagern allmählich die früheren exklusiven Treueverhältnisse.

In beiden Dokumenten, in "Inter Mirifica" und in der Uppsala-Erklärung, sind viele Fragen offen geblieben, und darin besteht die Chance beider Dokumente, in den ökumenischen Dialog einbezogen zu werden. Beide Gremien, Konzil und Vollversammlung, haben dokumentiert, daß die Massenkommunikationsmittel gottgegebene und damit verpflichtende Mittel sind. Beide Dokumente verpflichten dazu, den 
ökumenischen Dialog auch auf dem Gebiet der Medien intensiver zu führen als bisher und auf Grund beider Dokumente zu gemeinsam weiterführenden Erkenntnissen zu kommen. Der brennende Wunsch, daß die Medien dem wahrhaft Humanen dienen, „so daß wir als Blinde zu sehen beginnen und als Taube zu hören anfangen und daß wir als Sehende keine andere Wahl haben, als uns selber leidenschaftlich und sinnvoller im Leben der Welt Gottes zu engagieren",12 ist in beiden großen christlichen Gemeinschaften, dem Okumenischen Rat und der römisch-katholischen Kirche, für alle die, die mit den Massenmedien befaßt sind, nur noch dringlicher geworden.

\section{Anmerkungen:}

1. Otto B. Roegele in „Rheinischer Merkur“, Köln, vom 12. 7. 1968 (= 23. Jg.).

2. Karl Rahner, Herbert Vorgrimler: „Kleines Konzilskompendium“, (Freiburg im Breisgau 1966), S. 92.

3. Hanns Lilje "Kirche und Massenkommunikationsmittel" in: „Medium“, München, 5. Jg. 1968 , S. $1 \mathrm{ff}$.

4. Die Gedanken Marshall McLuhans („Die magischen Kanäle“, Düsseldorf-Wien 1968) sollten bei Formulierungen der Uppsala-Erklärung, die auf Entwicklungstendenzen zielen, im Hintergrund gesehen werden. Nach McLuhan befindet die Menschheit sich heute in einer radikalen Kommunikationsrevolution. Der "nachliterarische" Mensch taucht am Horizont auf. Die elektronischen Kommunikationsmittel stehen im Begriff, das geschriebene und gedruckte Wort als Hauptmedien unserer Kommunikation zu überrunden. Für viele Menschen der Erde - auch solche, die noch Analphabeten sind - sind bereits Hörfunk und Fernsehen zur Hauptinformationsquelle für politische, wirtschaftliche und kulturelle Ereignisse jenseits ihrer eigenen Erfahrung geworden, wobei Zeitungen und Bücher nur noch eine Hilfsrolle spielen. Millionen erwachsener Analphabeten in Asien, Afrika, Lateinamerika und Ozeanien kommen heute durch Ausbreitung von Hörfunk und Fernsehen aus dem vorliterarischen unmittelbar in das nachliterarische Zeitalter, ohne $\mathrm{d}_{a s}$ dazwischen liegende literarische Zeitalter zu durchlaufen. Der Wandel in der Art unserer Wirklichkeitserfahrung durch Entwicklung der elektronischen Kommunikationsmittel ist in der Geschichte der Menschheit allein dem Wandel vergleichbar, der eintrat, als die Entwicklung der Schreib- und später der Druckkunst es dem Menschen ermöglichte, mittels Buchstaben zu kommunizieren. (Vgl. Harvey Cox: "Stirb nicht im Warteraum der Zukunft", Stuttgart 1968, S. $41 \mathrm{ff}$.). "Inter Mirifica“ kann der Vorwurf nicht erspart bleiben, daß ihm jeder Hinweis auf Entwicklungstendenzen, die sich abzeichnen, fehlt.

5. Lexikon für Theologie und Kirche, Das Zweite Vatikanische Konzil, Konstitutionen, Dekrete, Erklärungen, Teil I (Freiburg im Breisgau 1966), S. 118 (Erläuterungen von Karlheinz Schmidthüs).

6. ebenda.

7. Evangelischer Pressedienst (epd), Zentralausgabe vom 17. 8. 1968.

8. Vgl. Anm. 5. Es sollte jedoch nicht übersehen werden, daß Medien „eine starke demokratisierende Kraft" haben. Albert van den Heuvel "Theologie, Kommunikation und Massenmedien “ in „Medium“, 4. Jg. 1967, S. 158.

9. Vgl. Anm. 5 .

10. Vgl. Anm. 7.

11. Albert van den Heuvel, a.a.O., S. 161.

12. Penry Jones in der Einführung zu dem Dokument „Die Kirche und die Medien der Massenkommunikation" vor dem Plenum der 4. Vollversammlung des Okumenischen Rates der Kirchen in Uppsala im Juli 1968.

\section{S U M M A R Y}

A declaration on "The Church and the Media of Mass Communication" was presented to the Fourth General Assembly of the Ecumenical Council of Churches, held in Uppsala, 
Sweden, July 4th to 19 th, 1968. This declaration was approved and recommended by the Assembly in its essential features. Compared with the Vatican Council's Decree on the Instruments of Social Communication ("Inter mirifica“) it shows some correspondence in principal, emphasizing, however, different conditions of communication activities in various countries. The general attitude of the declaration is best characterized by self-criticism, when in fact the decree is stressing church authority in communication. Both documents take notice of the missionary feasibilities in communication. In contrast to the Roman Catholic decree the Uppsala declaration states the responsibility of the churches to provide free flow of communication in admitting free access to news. There is hope for ecumenical dialogue on problems of communication since many questions were left unanswered in both papers.

\title{
RES U MEN
}

A la 4. Reunión General del Consejo Ecuménico de Iglesias, celebrado en Uppsala del 4 al 19 de Julio de 1968, le fue presentado, entre otros una Declaración sobre la „Iglesia y los Medios de Comunicación Social ${ }^{\star}$. El contenido esencial de esta Declaración fue aceptado y recomendado por la Reunión General. Si comparamos esta Declaración con el Decreto Conciliar sobre los Medios de Comunicación Social („Inter mirifica"), descubriremos una concordancia en los rasgos fundamentales. No obstante, la Declaración de Uppsala se ocupa sobre todo de las diversas condiciones de la actividad periodîstica en los diferentes paîses. Su postura es autocrîtica, mientras que el Decreto Conciliar acentúa fuertemente la primacîa de la Iglesia frente al periodismo. Ambos documentos constatan la importancia misional de las actividades periodistîcas. En oposición al punto de vista católico romano, la Declaración de Uppsala acentúa expresamente la obligación de las Iglesias de dar a conocer libremente sus informaciones. Como en ambas declaraciones han quedado muchos problemas sin resolver, abiertos, existen fundadas esperanzas de que se abra un diálogo ecuménico sobre los problemas de Comunicación.

\section{Presse und Funk im Territorium von Papua und Neuguinea (II)}

\author{
von Franz-Josef Eilers
}

\begin{abstract}
Dem ersten Teil dieses Beitrags in Nr. 3 CS (S. 197-208) über die Presse im Territorium von Papua und Neuguinea folgen eine tabellarische Ubersicht der Zeitungen und Zeitschriften und eine Darstellung der Situation des Hörfunks.
\end{abstract}

\section{Ubersicht über Zeitungen und Zeitschriften in Papua und Neuguinea}

In der nachfolgenden Übersicht handelt es sich um den ersten Versuch einer Gesamtschau der Presse im Territorium Papua und Neuguinea. Die einzelnen Angaben beruhen auf eigenen Untersuchungen, die ergänzt wurden durch Angaben in den 